\title{
Quantitative analysis of TM lateral leakage in foundry fabricated silicon rib waveguides
}

\author{
Anthony P. Hope,${ }^{1}$ Thach G. Nguyen, ${ }^{1}$ Arnan Mitchell, ${ }^{1}$ and Wim Bogaerts,${ }^{2 \dagger}$ \\ ${ }^{1}$ ARC Centre of Excellence for Ultrahigh bandwidth Devices for Optical systems (CUDOS) \\ and School of Electrical and Computer Engineering, RMIT University, Melbourne, Australia \\ ${ }^{2}$ Photonics Research Group, Ghent University-IMEC, Department of Information Technology, \\ Center of Nano-and Biophotonics. 9000 Gent, Belgium, \\ ${ }^{\dagger}$ now also with: Luceda Photonics, Dendermonde, Belgium
}

\begin{abstract}
We show that thin, shallow ridge, silicon-oninsulator waveguides exhibiting lateral leakage behaviour can be designed and fabricated using a standard silicon photonic foundry platform. We analyse the propagation loss through observation of the transmitted TM polarized guided mode and TE polarized radiation and experimentally demonstrate that propagation losses as low as $0.087 \mathrm{~dB} / \mathrm{mm}$ can be achieved. This demonstration will open a new frontier for practical devices exploiting lateral leakage behaviour with potential applications in the fields of biosensing and quantum optics among others.
\end{abstract}

\section{INTRODUCTION}

The lateral leakage of the fundamental TM guided mode in shallow etched waveguides is a curious mechanism that promises to provide a new suite of tools for the photonic toolbox. This mechanism is useful in the development of integrated sensors [1], [2], polarizers [3], liquid crystal platforms [4] and a recently proposed waveguide switching network [5].

In 2007, Webster et al. [6] discovered a width dependent loss mechanism in silicon-on-insulator shallow ridge waveguides, where phase matching can occur between forward propagating TM guided modes and in-plane propagating TE slab modes (Fig. 1(a)). The magnitude of this coupling is controlled by interference effects (Figs. 1(b,c)). Certain waveguide widths coherently cancel the outward TE vectors and are coined 'magic' widths (Figs. 1(d,e)). Complete reinforcement ('antimagic') results in the strongest TE presence (Fig. 1(f)).

To realize these 'leaky' waveguides, it was necessary to achieve very shallow ridges using unusual silicon processing, wet etching and thermal oxidation [6]. Due to these nonstandard conditions, research into this area has either been purely theoretical, or limited to small run research applications. Further, some researchers have abandoned shallow etched waveguides altogether or operate them only in the TE regime [7] as the lateral leakage is poorly understood and difficult to control.

In this paper, we show that lateral leakage can be reliably and predictably achieved using standard silicon foundry fabrication. We observe the width dependent leakage loss by using the transmission of the TM mode and also observe the leaked TE radiation directly. Our observations match well with the predicted behaviour. This standard fabrication platform offers
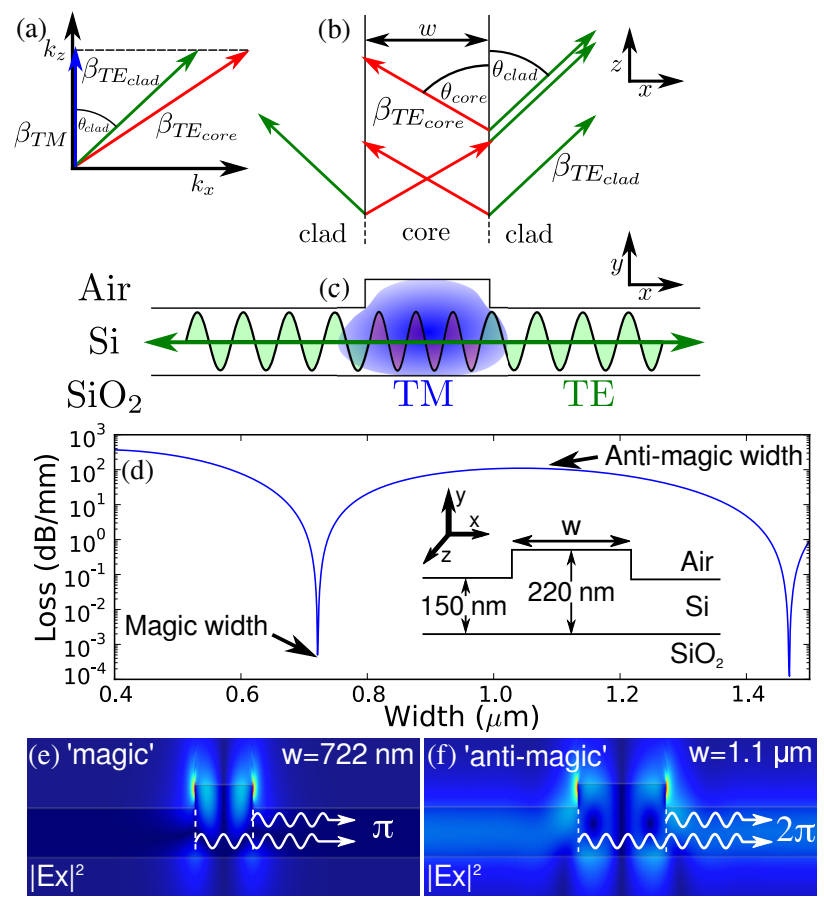

Fig. 1: The fundamental TM mode is altered and hybridized in shallow etched waveguides; (a) At certain in-plane angles, the radiative TE modes of the silicon slabs $\left(\beta_{T E_{c o r e}}\right.$ and $\left.\beta_{T E_{c l a d}}\right)$ can be phase matched to the fundamental guided TM mode $\left(\beta_{T M}\right)$, (b) interference is responsible for the appearance of 'magic' values where the radiation produced by either sidewall is interfering destructively, and this effect is related to the width (w) of the waveguide and the operational wavelength $(\lambda)$, (c) a representation of the mode profile of the hybridized TM-like mode containing both vertical and horizontal components, (d) the width dependence of the propagation loss of the fundamental TM mode, (e) at 'magic width' ( $\mathrm{w}=722 \mathrm{~nm}$ ) the lateral radiation cancels out and the waveguide has a low propagation loss. (f) at 'anti-magic width' ( $\mathrm{w}=1.1 \mu \mathrm{m})$ the radiation is reinforced and results in a strong field presence in the cladding.

benefits such as high precision, repeatability, throughput and the possibility of inclusion in multiple project wafer initiatives (MPW), lowering fabrication costs. This demonstration will improve the understanding and confidence in lateral leakage so that new and interesting devices can be developed and adopted into industrial integrated silicon photonic systems. Preliminary material from this work was published in [8]; however, here we present deeper analysis and substantial additional results. 

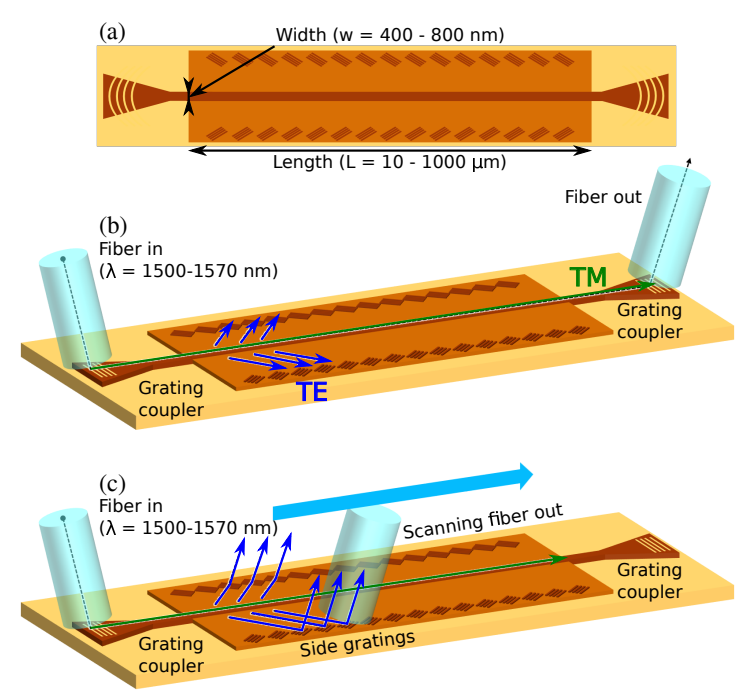

Fig. 2: (a) Schematic of the fiber measurement procedure for multiple defined waveguides with varying widths $(\mathrm{w}=400-800 \mathrm{~nm})$ and lengths $(\mathrm{L}=10-1000 \mu \mathrm{m})$, (b) measuring the transmission of $\mathrm{TM}$ light through the waveguides, (c) collecting the converted TE light received at the side gratings with a scanning fiber or imaged with an infrared camera.

\section{DESIGN OF THIN SHALLOW RIDGES FOR CMOS} FABRICATION AND GRATING COUPLER CHARACTERISATION

This section aims to show that thin shallow ridge waveguides exhibiting lateral leakage can be realized using standard silicon photonic fabrication. We chose the ePIXfab IMEC standard platform using $220 \mathrm{~nm}$ thick silicon on $2 \mu \mathrm{m}$ silicon dioxide, patterned with either $70 \mathrm{~nm}$ or full $220 \mathrm{~nm}$ etching with minimum features sizes of 150 and $130 \mathrm{~nm}$, respectively [9]. The upper cladding was air. The devices were designed and simulated using the 'IPKISS' framework [10] and a custom eigenmode expansion model [11] to enable simulation of the lateral leakage behaviour. Our simulations predicted that ridges with these parameters, should support a single TM mode at a wavelength of $1550 \mathrm{~nm}$ up to a width of about $850 \mathrm{~nm}$. Fig. 1(d) presents the predicted lateral leakage loss as a function of ridge width, clearly showing low-loss 'magic' width behaviour at a width of $722 \mathrm{~nm}$.

We designed a series of waveguides with a range of widths from $400-800 \mathrm{~nm}$ and lengths $(10 \mu \mathrm{m}-1 \mathrm{~mm})$. Each waveguide was interfaced to single mode fibers using tapered focussed TM grating couplers [12], [13] designed for optimum efficiency in the $\lambda=1500-1570 \mathrm{~nm}$ range. To observe the TE radiation produced by lateral leakage, a series of grating couplers, phase matched and oriented to align with the expected TE radiation, were included on either side of the waveguide along its length. Fig. 2(a) presents a schematic of the designed structure and Figs. 2(b-c) illustrate the methods for measuring the TM waveguide transmission and the generated TE lateral leakage radiation, respectively.

The designed devices were fabricated, and to determine the actual waveguide dimensions, a sample of devices were crosssectioned and imaged using scanning electron microscopy (SEM). An SEM image of one of the realized structures is presented in Fig. 3. The silicon thickness was found to be 215 $\mathrm{nm}$, the ridge was $70 \mathrm{~nm}$ and the ridge widths tended to be $10-20 \mathrm{~nm}$ wider than the nominal design.

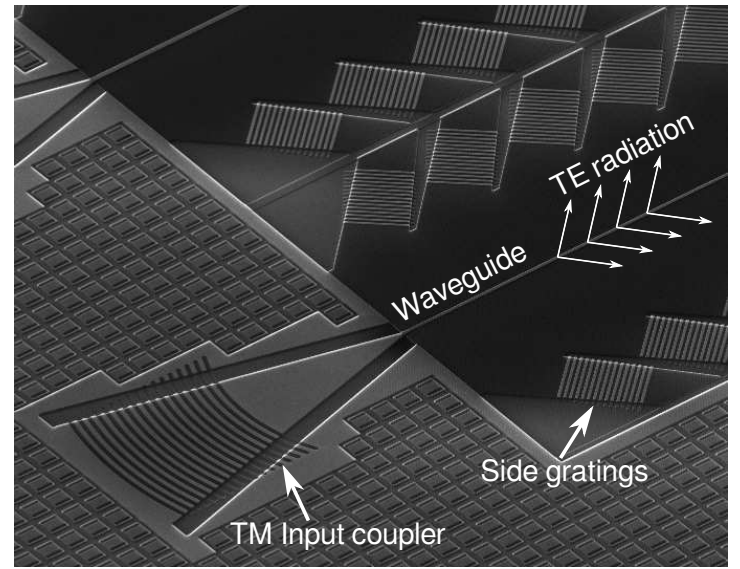

Fig. 3: Scanning electron microscope image of the input section of our fabricated waveguides, showing the input focussed TM coupler, waveguide and side TE gratings. All outer regions have been completely etched to isolate each individual waveguide from another.
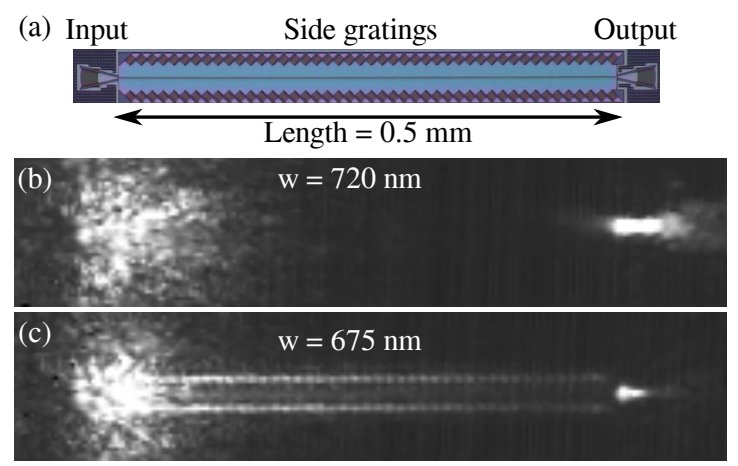

Fig. 4: (a) Optical microscope image of a fabricated waveguide of length $0.5 \mathrm{~mm}$, including input, output and side grating couplers, (b) infrared imaging of a $720 \mathrm{~nm}$ wide waveguide which is close to the predicted 'magic' low loss condition, (c) infrared imaging of a 675 nm wide 'non-magic' waveguide demonstrates how light is converted to TE radiation away from this resonance.

Simulating with the slab thickness at $215 \mathrm{~nm}$, we found that the predicted 'magic' width is $717 \mathrm{~nm}$ at a wavelength of 1550 $\mathrm{nm}$. From this point, all reported dimensions are as-fabricated.

To qualitatively test the structures, an infrared camera was positioned above the $0.5 \mathrm{~mm}$ long waveguides and the input coupler was excited with a wavelength of $1550 \mathrm{~nm}$. Fig. 4(a) shows a visible wavelength microscope image of one of the waveguide structures. Fig. 4(b) shows the infrared image of the $720 \mathrm{~nm}$ wide waveguide excited at $1550 \mathrm{~nm}$. On the input side we see scattered light. On the output side we see a very bright spot corresponding to light exiting through the output TM grating coupler. In between we see darkness indicating that light is confined within the waveguide.

Fig. 4(c) shows an infrared camera image of the $675 \mathrm{~nm}$ wide waveguide excited at $1550 \mathrm{~nm}$. Again we see scattered light from the input. The light at the output TM grating coupler is dimmer than in Fig. 4(c). In between we see excitation of the TE side gratings, decreasing with distance from the input. Fig. 4(c) presents clear evidence of lateral leakage behaviour in thin-ridge waveguides while Fig. 4(b) presents clear evidence of the suppression of lateral leakage when the waveguide is 'magic' width. Together these represent the first evidence of lateral leakage observed in mass fabricated silicon waveguides. 


\section{WAVEGUIDE CHARACTERIZATION}

\section{A. Width dependent propagation loss}

We first quantify the propagation loss as a function of waveguide width using the cut-back method, measuring the transmission efficiency for identical waveguides of different lengths and then extract the loss per unit length. The transmission of waveguides of widths 420, 720, 760 and $820 \mathrm{~nm}$ and lengths between 10 and $1000 \mu \mathrm{m}$ were each measured at a wavelength of $1550 \mathrm{~nm}$ and the transmission efficiency as a function of length is plotted in Fig. 5. To eliminate the response of the grating couplers, a fully etched wire waveguide of width $420 \mathrm{~nm}$ with identical grating couplers was used as a normalization reference. The propagation loss $(\alpha)$ is the slope of the line in $\mathrm{dB} / \mathrm{mm}$. These results clearly show that there is a strong dependence of observed loss on waveguide width with $\alpha=0.357 \mathrm{~dB} / \mathrm{mm}$ when $\mathrm{w}=720 \mathrm{~nm}$ rising to $\alpha=585.95$ $\mathrm{dB} / \mathrm{mm}$, similar to the behaviour reported in [6].

\section{B. Wavelength dependent propagation loss}

Lateral leakage is a resonant effect and thus we expect the loss to not only depend on ridge width, but also on wavelength [6]. For $1550 \mathrm{~nm}$, we expect 'magic' width behaviour, where the lateral leakage loss is completely cancelled, at a width of $717 \mathrm{~nm}$. To characterize the wavelength dependent propagation loss, we measured the power transmitted through waveguides with widths of $675,700,720$ and $740 \mathrm{~nm}$ over the entire fabricated length range, while scanning the wavelength from 1500-1570 nm as illustrated in Fig. 2(b). The loss of each waveguide width was again determined using a linear fit.

Fig. 6 presents the transmitted power per unit length for each waveguide as a function of wavelength. Resonant loss cancellation is clearly evident with waveguide widths of 700 $\mathrm{nm}$ and $720 \mathrm{~nm}$ exhibiting minimal loss of $0.238 \mathrm{~dB} / \mathrm{mm}$ and $0.087 \mathrm{~dB} / \mathrm{mm}$ at wavelengths of $1500 \mathrm{~nm}$ and $1563 \mathrm{~nm}$, respectively.

The expected wavelength dependent lateral leakage behaviour was simulated using eigenmode expansion [11] and these predictions are also presented in Fig. 6. An excellent match to the measured values is evident, particularly the 720 $\mathrm{nm}$ width waveguide with transmission loss approaching zero at the resonant wavelength.

\section{Conversion of TM guided propagation to TE radiation}

To observe the TE radiation we use side gratings, with period and orientation for efficient diffraction of the TE mode propagating at the expected angle of 45 degrees, as illustrated in Fig. 2(c). Waveguides of fabricated widths $w=675,720$ and $810 \mathrm{~nm}$ and length $500 \mu \mathrm{m}$ were excited with $\lambda=1550 \mathrm{~nm}$ and the intensity measured at the TE side gratings as a function of length is presented in Fig. 7. The rapid oscillations are due to the numerous, discrete side gratings. A linear fit to each trace indicates exponential decay with the slope yielding the the attenuation coefficient. These are similar to those of Fig. 6 indicating that the observed width dependent loss is due to conversion of the TM guided light into lateral TE radiation.

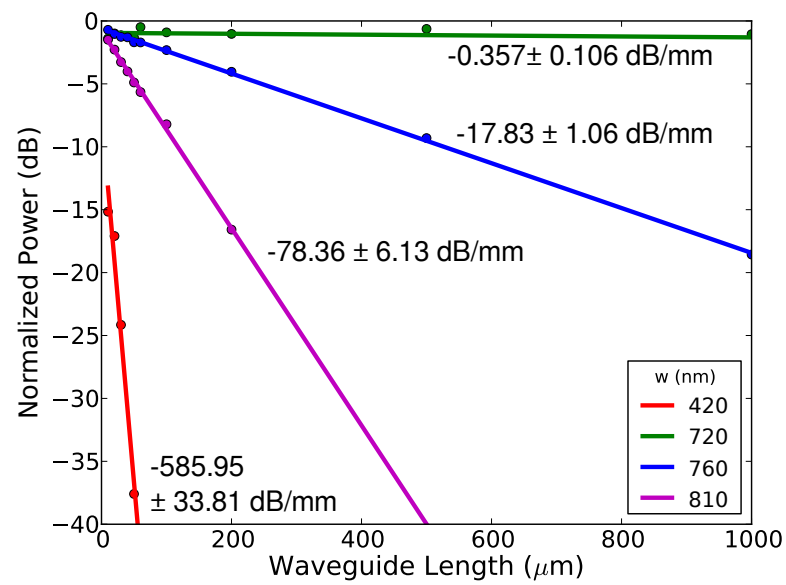

Fig. 5: TM transmission at $\lambda=1550 \mathrm{~nm}$ vs waveguide length for different waveguide widths. The gradient yields the waveguide propagation loss $(\alpha)$.

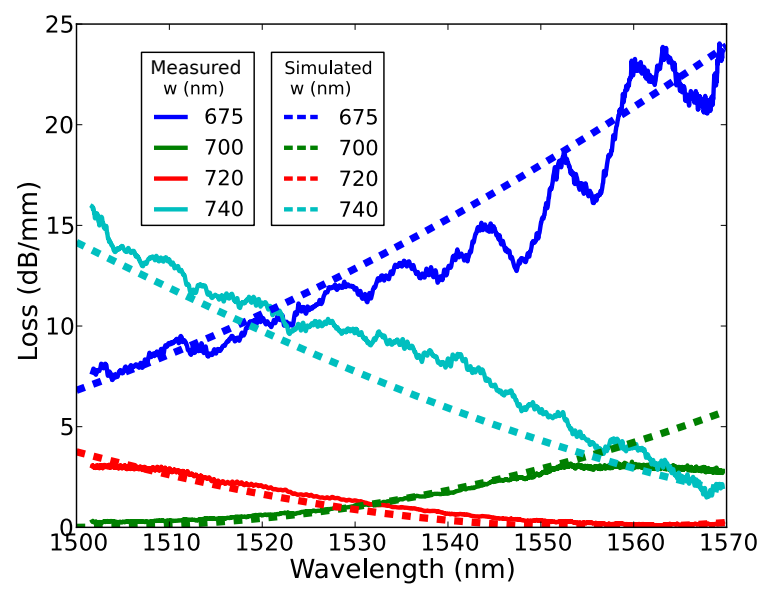

Fig. 6: The wavelength dependant TM transmission loss of four fabricated waveguide widths. Simulations have been performed to compare the spectral response of each waveguide width.

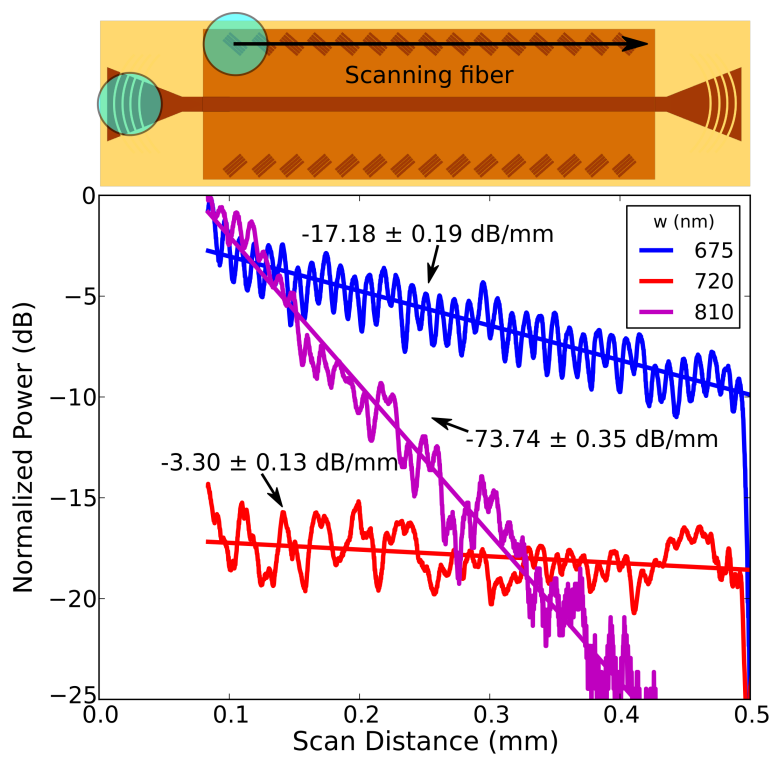

Fig. 7: Scanning an output fiber across the center of these side gratings provides quantitative information regarding the TE conversion and the loss can be estimated with a linear fit. 


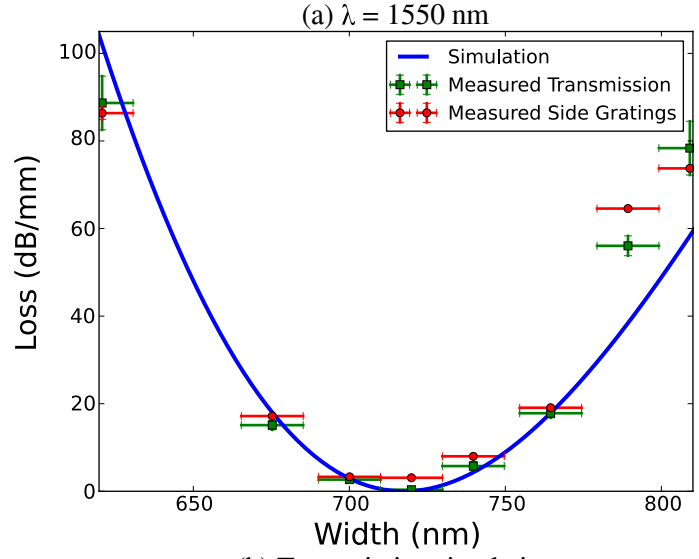

(b) Transmission simulation
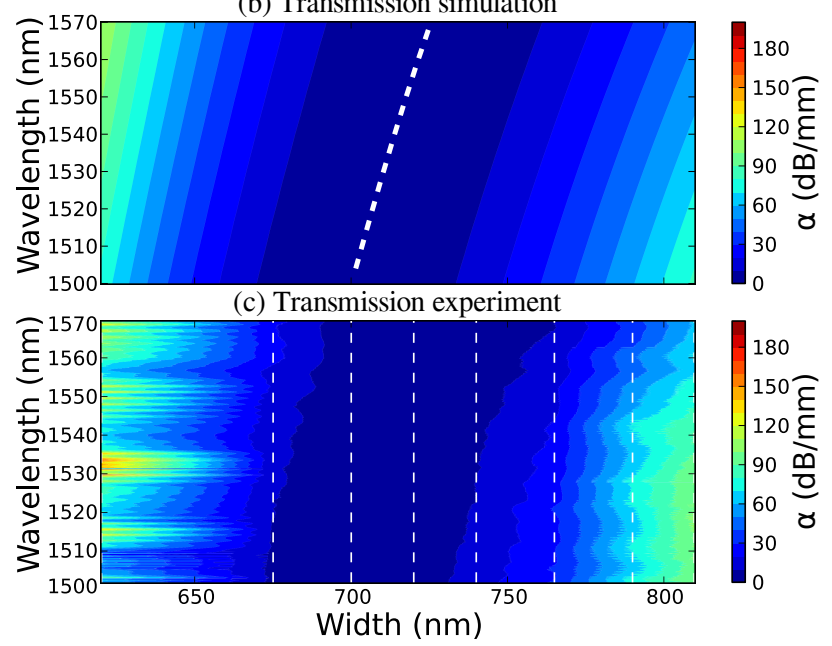

Fig. 8: (a) Experimental propagation loss obtained from the transmission measurements and side grating measurements, compared to the simulated loss at $\lambda=1550 \mathrm{~nm}$. (b) Simulation of the propagation loss $(\alpha)$ expected for $215 \mathrm{~nm}$ high, $70 \mathrm{~nm}$ etched air clad SOI waveguides as a function of waveguide width and wavelength, the ideal magic width is dashed white, (c) Propagation loss $(\alpha)$ calculated from the interpolated experimental transmission data, which is in good agreement with the simulation.

\section{ANALYSIS OF LEAKAGE MEASUREMENTS}

We have characterized the fabricated thin ridge waveguides over a wide range of wavelengths, widths and lengths, and we have compared these measurements with simulations based on the measured as-fabricated waveguide parameters. Cut-back analysis of the direct TM transmission loss (Fig. 5) and the observation of the TE radiation (Fig. 7) was used to extract the propagation loss. Fig. 8(a) presents a survey of the propagation loss as a function of waveguide width at $\lambda=1550 \mathrm{~nm}$ extracted from both the measured TM transmission and TE radiation. The vertical and horizontal error bars represent uncertainty in the measured loss and dimensional accuracy $( \pm 10 \mathrm{~nm})$, respectively. The predicted loss of the fundamental waveguide mode due solely to TE radiation, is also presented in Fig. 8(a). Excellent agreement between both measurements and prediction is evident up to widths of $760 \mathrm{~nm}$. At widths above $780 \mathrm{~nm}$, the waveguide is expected to begin to support a strongly radiating higher order resonance, which becomes a guided mode at $850 \mathrm{~nm}$, and this may explain the additional transmission loss observed at these widths and above.

A wavelength sweep was also conducted during the mea- surements. Fig. 8(b) and (c) present the predicted and interpolated, experimentally measured propagation loss as a function of waveguide width and $\lambda$, respectively. Again we find a very good match, with a slight skew towards the higher waveguide widths.

\section{CONCLUSION}

We have shown that thin, shallow-etched SOI waveguides exhibiting lateral leakage behaviour can be realized using standard, silicon photonic foundry fabrication. We have characterized the waveguides via analysis of the transmitted TM mode and also direct observation of the TE radiation. We find very good correspondence between eigenmode expansion predictions and two different measurement procedures. Magic width behaviour with resonant cancellation of the leakage leading to losses as low as $0.087 \mathrm{~dB} / \mathrm{mm}$ have been observed experimentally. The ability to design, simulate and experimentally realize these passive waveguide structures using a standard silicon photonic fabrication platform creates new opportunities for practical devices exploiting lateral leakage with potential applications including biophotonic sensing and quantum information processing.

\section{REFERENCES}

[1] T. G. Nguyen, R. S. Tummidi, T. L. Koch, A. Mitchell, "Lateral leakage of TM-like mode in thin-ridge Silicon-on-Insulator bent waveguides and ring resonators," Opt. Express, 18, 7243-7252 (2010).

[2] R. Hainberger, R. Bruck, P. Muellner, K. Roppert, W. Boxleitner, C. Pacher, and N. Finger, "Design of silicon and polymer photonic waveguide structures for sensing applications," Proc. SPIE Photonics North, 73862Q (2009).

[3] D. Dai, Z. Wang, N. Julian, and J. Bowers, "Compact broadband polarizer based on shallowly-etched silicon-on-insulator ridge optical waveguides," Opt. Express 18, 27404-27415 (2010).

[4] T. Ako, J. Beeckman, W. Bogaerts, and K. Neyts, "Tuning the lateral leakage loss of TM-like modes in shallow-etched waveguides using liquid crystals," Applied Optics, 53(2), 214-220 (2014).

[5] A. Hope, T. Nguyen, A. Greentree, and A. Mitchell, "Long-range coupling of silicon photonic waveguides using lateral leakage and adiabatic passage," Opt. Express 21, 22705-22716 (2013).

[6] M. Webster, R. Pafchek, A. Mitchell, and T. Koch, "Width dependence of inherent TM-mode lateral leakage loss in silicon-on-insulator ridge waveguides," IEEE Photon. Technol. Lett. 19(6), 429-431 (2007).

[7] L. Jin, M. Li, J. He, "Highly-sensitive silicon-on-insulator sensor based on two cascaded micro-ring resonators with vernier effect," Opt. Comm., 284(1), 156-159 (2011).

[8] A. P. Hope, T. G. Nguyen, W. Bogaerts, and A. Mitchell. "Experimental Demonstration of TM Lateral Leakage in a Standard SOI Photonics Platform," Group IV Photonics 2014, pp. 77-78 (2014).

[9] S.K. Selvaraja, P. Jaenen, W. Bogaerts, D. Van Thourhout, P. Dumon, and R. Baets, "Fabrication of Photonic Wire and Crystal Circuits in Siliconon-Insulator Using 193-nm Optical Lithography," J. Lightwave Technol., 27 4076-4083 (2009).

[10] M. Fiers, E. Lambert, S. Pathak, B. Maes, P. Bienstman, W, Bogaerts, and P. Dumon, "Improving the design cycle for nanophotonic components," J. of Comput. Sci., 4, 313-324 (2013).

[11] T. Nguyen, R. Tummidi, T. Koch, and A. Mitchell, "Rigorous modeling of lateral leakage loss in SOI thin-ridge waveguides and couplers," Photon. Technol. Lett., IEEE 21, 486-488 (2009).

[12] F. Van Laere, T. Claes, J. Schrauwen, S. Scheerlinck, W. Bogaerts, D. Taillaert, L. O'Faolain, D. Van Thourhout, and R. Baets, "Compact focusing grating couplers for silicon-on-insulator integrated circuits," IEEE Photon. Technol. Lett., 1923 1919-1921 (2007).

[13] D. Vermeulen, K. Van Acoleyen, S. Ghosh, S. Selvaraja, W.A.D. De Cort, N.A Yebo, E. Hallynck, K. De Vos, P.P.P. Debackere, P. Dumon, W. Bogaerts, G. Roelkens, D. Van Thourhout, and R. Baets "Efficient Tapering to the Fundamental Quasi-TM Mode in Asymmetrical Waveguides," ECIO (2010) 